\title{
Optimization of the preparation process of rice quinoa gel by response surface methodology
}

\author{
Zhou Yilun ${ }^{1, \mathrm{a}}$, Feng Xiaoguang ${ }^{2, \mathrm{~b}}$, Chen Xiangning*, Zhao Fushi ${ }^{3, \mathrm{C}}$ \\ 1. 3 Food Science and Engineering College, Beijing University of Agriculture, Beijing 102206, China; \\ ${ }^{2}$ Beijing Yunong High Quality Cultivation of Agricultural Products Company, Beijing 101400, China \\ ${ }^{*}$ Food Science and Engineering College, Beijing University of Agriculture, Beijing 102206, China;
}

\begin{abstract}
Rice noodles is a common food, but it is faced with the characteristics of hard texture and poor nutrition after aging. Quinoa is rich in dietary fiber and nutritious and can enrich the color and taste of rice noodles. In this paper, red quinoa is used as an additive, gelatinization time, quinoa added amount, and water added are selected as influencing factors on a single factor basis, and the hardness of rice quinoa gel is used as the response value to carry out experiments to optimize rice quinoa gelling Glue production process. The results show that the best process is: gelatinization time $30 \mathrm{~min}$, quinoa addition amount $30 \%$, material to water ratio $1: 2(\mathrm{~g} / \mathrm{mL})$. The test product of this formula has uniform color, uniform gel, strong aroma, good taste and fine mouthfeel.
\end{abstract}

\section{Introduction}

Rice is a very common staple food. In 2016, the Food and Agriculture Organization of the United Nations estimated that about 740 million tons of rice were harvested worldwide, of which about $90 \%$ (about 670 million tons) of rice was produced and consumed in Asia. As one of the staple foods of people, rice is often cooked and processed for consumption. The main carbohydrate in rice is rice starch, which mainly exists in endosperm cells and accounts for $90 \%$ of the dry weight of rice[1]. But obviously, cooked rice is prone to ageing during storage. And the aging of starch will make the taste and texture of starch-based foods worse, shorten the shelf life and even cause slightly lower digestion and absorption rate. Therefore, inhibiting the aging of starch is very critical to maintaining the quality of starch-based foods.

Quinoa (Chenopodium quinoa Willd.) is a food crop native to the Andean plateau of South America. It is also a traditional staple food of the Incas. It is also the only food recognized by FAO that a single plant can meet the basic nutritional needs of the human body. As quinoa has the advantages of salt-alkali tolerance and high altitude adaptability, it has been introduced to many high-altitude areas in my country and is considered to be a promising alternative crop $^{[2-3]}$.

Quinoa grains are rich in high-quality protein and have a balanced amino acid ratio, which can meet human's essential amino acid requirements and does not contain gluten $^{[4]}$. In addition, quinoa grains are rich in fatty acids, which are higher than common grains. Studies have shown that the fatty acids in quinoa seeds are mainly unsaturated fatty acids such as linoleic acid, linoleic acid and oleic acid, with a content of more than $80 \%$; the ratio of linoleic acid to linolenic acid (LA/ALA) in its fatty acids is 6.2, which is in line with FAO /WHO recommended value ${ }^{[5]}$. Most importantly, quinoa itself is rich in dietary fiber, with a total content of $13.4 \%$, of which soluble fiber is 2.4\% (Ruales, 1994; Lisa M Lamothe, 2015). Dietary fiber can reduce blood sugar level and low-density lipoprotein cholesterol content and maintain cardiovascular and cerebrovascular health, which is very important for diabetic patients. Moreover, due to its strong water absorption capacity, it will produce a feeling of fullness after ingestion, resulting in reduced food intake, so it can To weight loss. Dietary fiber is also a polysaccharide, and it also has the effect of delaying the aging of starch. Adding it to rice starch can enrich the nutrients and delay the aging trend of rice starch.

This article screened the types of quinoa additives. On the basis of single factor, the response surface optimization method was used to study the key factors influencing the production process of rice gel with red quinoa as an additive.

\section{Materials and methods}

\subsection{Materials}

The rice comes from Yufurong Thai Fragrant Rice; the three colors of quinoa are from Qilian Farm's organic quinoa. The water bath and other instruments came from the Food Laboratory of Beijing Agricultural College.

\footnotetext{
*Corresponding author's e-mail: cxn@bua.edu.cn
} 


\section{2 method}

\subsubsection{Preparation of rice quinoa gel}

Break the rice with a pulverizer, pass the powdered rice through a 100-mesh sieve, weigh a certain amount of rice flour into a beaker, add different amounts of red quinoa powder that has passed through the 100-mesh sieve), press the rice flour and water Add distilled water at a ratio of 1:2 (m: V). After stirring uniformly, heat it in a water bath at $95^{\circ} \mathrm{C}$ for 30 minutes. When heating starts, keep stirring with a glass rod for 2 minutes, and then cover with plastic wrap to prevent excessive evaporation of water. Finally, the gelatinized rice gel is sealed with plastic wrap and placed in a refrigerator at $4^{\circ} \mathrm{C}$ for storage.

\begin{tabular}{|c|c|c|c|c|c|c|c|}
\hline 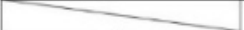 & Scoring item & & & & & & \\
\hline project & Grading rules & 1 & 2 & 3 & 4 & 5 & 6 \\
\hline \multirow{4}{*}{ Appearance ( 10 points) } & The color is attractive, the surface is smooth, I like it very much (10-9points) & & & & & & \\
\hline & The color is brighter, the surface is relatively flat, I like it better ( $8-6$ points) & & & & & & \\
\hline & Flatness and color are average, neither like nor dislike (5-3 points) & & & & & & \\
\hline & Rough surface and poor color, I don't like it (2-1 points) & & & & & & \\
\hline \multirow{4}{*}{ Flavor (10 points) } & Coordinated aroma of quinoa and rice ( $10-9$ points) & & & & & & \\
\hline & Has a more harmonious aroma of quinoa and rice ( $8-6$ points) & & & & & & \\
\hline & Quinoa has a strong or light flavor, not very harmonious (5-3 points) & & & & & & \\
\hline & The qquinoa flavor is too strong or too weak, inconsistent (2-1 points) & & & & & & \\
\hline \multirow{4}{*}{ Taste (10 points) } & The taste is coordinated, just right, and very elastic (10-9 points) & & & & & & \\
\hline & Slightly soft or dry, good elasticity (8-6 points) & & & & & & \\
\hline & More water soft or dry hard, average elasticity (5-3 points) & & & & & & \\
\hline & The water is soft or dry and hard, and the elasticity is poor (2-1 points) & & & & & & \\
\hline & total & & & & & & \\
\hline
\end{tabular}

Fig 1. Sensory Evaluation Form of Rice Quinoa Gel
Fig 1 is the sensory evaluation table. Ten people ( 5 males and 5 females) who have undergone sensory evaluation training are selected to score the rice quinoa gel according to the evaluation table.

\subsubsection{Single factor experiment}

After red quinoa replaces part of the rice flour, the taste of quinoa rice gel mainly depends on three factors: gelatinization time, water ratio and quinoa addition amount. In order to optimize the taste and also serve as a better aging research object, a single factor experiment was performed. Keep other factors unchanged, gelatinization time $25-50 \mathrm{~min}$, quinoa addition amount 0 $50 \%$, and water amount 1.4-2.4 times for single factor test.

Table 1. Single factor test factor levels

\begin{tabular}{ccccccc}
\hline factor & \multicolumn{7}{c}{ Level } \\
\hline $\begin{array}{c}\text { Gelatinization } \\
\text { time (min) }\end{array}$ & 25 & 30 & 35 & 40 & 45 & 50 \\
$\begin{array}{c}\text { Add water } \\
\text { amount (g) }\end{array}$ & 14 & 16 & 18 & 20 & 22 & 24 \\
$\begin{array}{c}\text { Quinoa added } \\
\text { amount (g) }\end{array}$ & 0 & 1 & 2 & 3 & 4 & 5 \\
\hline
\end{tabular}

\subsubsection{Screening of Quinoa Species}

The common quinoa on the market generally comes in three colors, namely red, white and black. Or a product with a mixture of three colors. However, the aroma of the three types of quinoa after gelatinization is not the same as the color of rice products. In order to screen out which color of quinoa is most suitable for making rice quinoa gel, a preliminary sensory evaluation experiment was carried out. Ten people $(5$ males and 5 females, all trained in sensory evaluation) were selected for sensory evaluation of different quinoa and rice gel.

\subsubsection{Sensory evaluation}

\subsubsection{Hardness determination of texture analyzer}

After the above-mentioned rice quinoa gel was cooled to room temperature, it was placed on a texture analyzer to test the hardness of the rice quinoa gel. Use TA10 probe, the speed before test is $1.0 \mathrm{~mm} / \mathrm{s}$, the test speed is $1.0 \mathrm{~mm} / \mathrm{s}$, and the speed after test is $1.0 \mathrm{~mm} / \mathrm{s}$. The compression distance is $5 \mathrm{~mm}$, the trigger force is $5 \mathrm{~g}$, and the data acquisition rate is set to 200pps. Record the hardness and use it as a dependent variable during optimization.

\subsubsection{Response surface experiment}

The experiment was designed according to the BoxBehnken central combination design principle, with the elasticity of the final product as the dependent variable, gelatinization time, quinoa addition, and water addition ratio as independent variables, optimized with DesignExpert 8.0.6.1. 


\section{Results \& Discussion}

\subsection{Pre-experiment results}

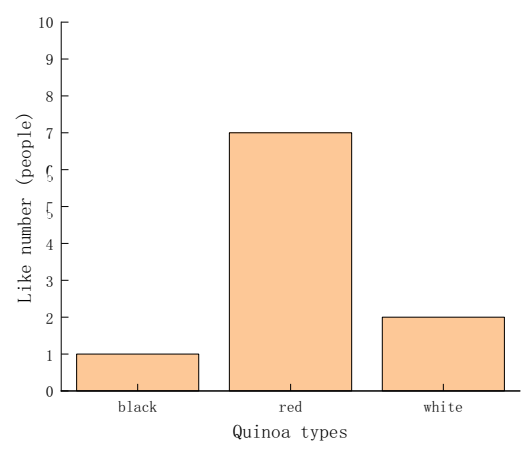

Fig 2. Add the number of preferences for different types of quinoa

It can be seen from Fig 2 that the number of people who love red quinoa accounts for $70 \%$ of the total, indicating that red quinoa is more in line with the taste of the public, so follow-up experiments use red quinoa to add rice gel. 3.2 Single factor experiment results

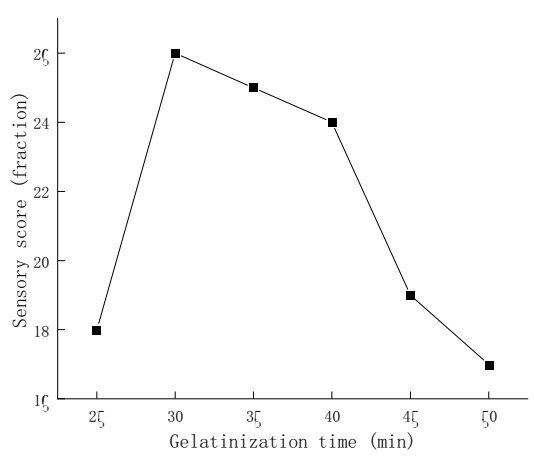

Fig 3. The effect of different gelatinization time on the sensory score of rice gel

It can be seen from Fig 3 that when the gelatinization time is 30 minutes, the sensory score is greatly improved compared to 25 minutes, reaching a peak of 26 points. The score gradually decreased after 30 minutes. After more than 40 minutes, it drops sharply; from Fig 4, it can be seen that the peak value of 24 points is reached when the amount of water is doubled, and the scores at both ends of the top change greatly. Fig 5 shows that when quinoa is added at $3 \mathrm{~g} / 10 \mathrm{~g}$, the sensory score is up to 26 points. When quinoa is added from $0 \mathrm{~g}$ to $3 \mathrm{~g}$, the sensory score is greatly affected, and the score decreases slowly when it exceeds $3 g$.

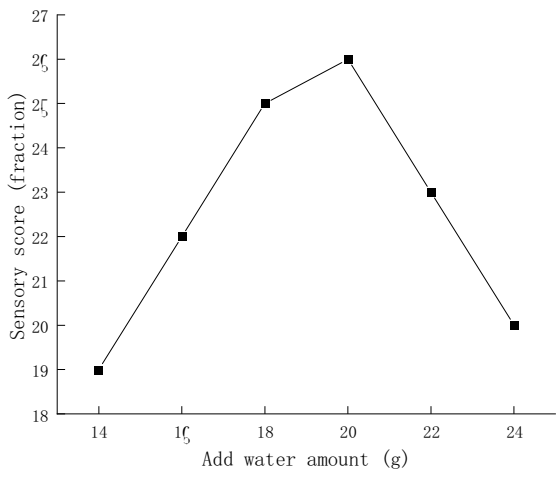

Fig 4. The influence of different water addition on the sensory score of rice gel

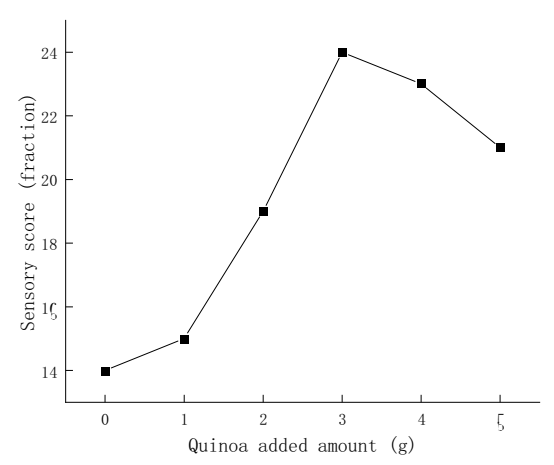

Fig 5. The effect of different quinoa on the sensory score of rice gel

\subsection{Experimental results of response surface optimization}

Table 2. Response surface experimental design factor level coding table

\begin{tabular}{ccccc}
\hline Experimental factors & $\begin{array}{c}\text { Coded } \\
\text { value }\end{array}$ & \multicolumn{3}{c}{$\begin{array}{c}\text { Factor level and } \\
\text { coding }\end{array}$} \\
\cline { 3 - 5 } & & -1 & 0 & 1 \\
\hline $\begin{array}{c}\text { Gelatinization time } \\
\text { (min) }\end{array}$ & $\mathrm{A}$ & 25 & 30 & 35 \\
$\begin{array}{c}\text { Quinoa added amount } \\
\text { (g) }\end{array}$ & $\mathrm{B}$ & 2 & 3 & 4 \\
Add water amount (g) & $\mathrm{C}$ & 18 & 20 & 22 \\
\hline
\end{tabular}


Table 3. Box-Behnken experiment and results

\begin{tabular}{ccccc}
\hline Test Number & A & B & C & Hardness(g) \\
\hline 1 & 35 & 4 & 20 & 435 \\
2 & 25 & 4 & 20 & 525 \\
3 & 35 & 3 & 22 & 412 \\
4 & 30 & 3 & 20 & 343 \\
5 & 35 & 3 & 18 & 570 \\
6 & 30 & 3 & 20 & 345 \\
7 & 30 & 3 & 20 & 340 \\
8 & 25 & 2 & 20 & 490 \\
9 & 25 & 3 & 22 & 363 \\
10 & 35 & 2 & 20 & 600 \\
11 & 30 & 3 & 20 & 348 \\
12 & 25 & 3 & 18 & 626 \\
13 & 30 & 4 & 22 & 438 \\
14 & 30 & 2 & 22 & 472 \\
15 & 30 & 4 & 18 & 631 \\
16 & 30 & 2 & 18 & 700 \\
17 & 30 & 3 & 20 & 342 \\
\hline
\end{tabular}

\subsubsection{Analysis of variance}

Using Design-Expert 8.0.6.1 fitting analysis, the resp onse surface model quadratic multiple regression equ ation is obtained as follows: Hardness $=343.60+1.63 \mathrm{~A}$ $-29.12 \mathrm{~B}-105.25 \mathrm{C}-50.00 \mathrm{AB}+26.25 \mathrm{AC}+8.75 \mathrm{BC}+50.70 \mathrm{~A}^{2}$ $+118.20 \mathrm{~B}^{2}+98.45 \mathrm{C}^{2}$.

The results of the analysis of variance are shown in the table. It can be seen that $\mathrm{P}<0.001$, which indicates that the regression effect of the regression model is significant, and the lack of fit term is not significant (P0.0507>0.05), indicating that the model can better describe the relationship between each factor and the response value Relationship, this regression equation can be used to determine the optimal process. The model $\mathrm{R}^{2} \mathrm{adj}$ is 0.9978 , indicating that the model can explain about $99.78 \%$ of the response value change; the regression model retest coefficient R is R $2=99.90 \%$, and the correlation is good, the corrected retest coefficient R2adj is $98.71 \%$, the test value and correction The value difference is small, indicating that the model is better. The precision Adeq Precision is $83.779>4$, indicating that the model precision is reasonable; the coefficient of variation C.V is $1.19 \%<10 \%$, indicating that the test value is close to the predicted value, the experimental reliability and accuracy are high, and the experimental error is small.

It can be seen from the table that the primary factors $\mathrm{B}$ and $\mathrm{C}$; the $\mathrm{P}$ values of the interaction terms $\mathrm{AB}$ and $\mathrm{AC}$ are all less than 0.01 , and the results are extremely significant; the $\mathrm{P}$ value of the interaction term $\mathrm{BC}$ is 0.0167 while greater than 0.01 and less than 0.05 , and the results are significant; $\mathrm{A}$. The $\mathrm{P}$ values of the quadratic terms $\mathrm{A}^{2}, \mathrm{~B}^{2}$ and $\mathrm{C}^{2}$ are greater than 0.05 , and the result is not significant. According to the $\mathrm{F}$ value, the order of influence on hardness is $\mathrm{C}$ (water addition)> B (quinoa addition) $>$ A (gelatinization time). The results of the analysis of variance are shown in Table

Table 4. Box-Be hnken test variance analysis

\begin{tabular}{|c|c|c|c|c|c|c|}
\hline $\begin{array}{l}\text { Source of } \\
\text { Variation }\end{array}$ & $\begin{array}{l}\text { Sum of } \\
\text { Square }\end{array}$ & df & MS & $\mathbf{F}$ & $\mathbf{P}$ & $\begin{array}{c}\text { Significa } \\
\text { nce }\end{array}$ \\
\hline Model & 230200.00 & 9 & 25582.74 & 816.04 & $<0.0001$ & $\begin{array}{c}\text { Significa } \\
n t\end{array}$ \\
\hline $\mathbf{A}$ & 21.13 & 1 & 21.13 & 0.67 & 0.4388 & \\
\hline B & 6786.12 & 1 & 6786.12 & 216.46 & $<0.0001$ & $* *$ \\
\hline C & 88620.50 & 1 & 88620.50 & 2826.81 & $<0.0001$ & $* *$ \\
\hline $\mathbf{A B}$ & 10000.00 & 1 & 10000.00 & 318.98 & $<0.0001$ & $* *$ \\
\hline AC & 2756.25 & 1 & 2756.25 & 87.92 & $<0.0001$ & $* *$ \\
\hline BC & 306.25 & 1 & 306.25 & 9.77 & 0.0167 & $*$ \\
\hline $\mathbf{A}^{2}$ & 10823.12 & 1 & 10823.12 & 345.23 & 345.23 & \\
\hline $\mathbf{B}^{2}$ & 58826.27 & 1 & 58826.27 & 1876.44 & 1876.44 & \\
\hline $\mathbf{C}^{2}$ & 40810.12 & 1 & 40810.12 & 1301.76 & 1301.76 & \\
\hline Residual & 219.45 & 7 & 31.25 & & & \\
\hline Lack of Fit & 182.15 & 3 & 60.75 & 6.53 & 0.0507 & $\begin{array}{c}\text { Not } \\
\text { Significa } \\
\text { nt }\end{array}$ \\
\hline Pure Error & 37.12 & 4 & 9.30 & & & \\
\hline Cor Total & 230500.00 & 16 & & & & \\
\hline
\end{tabular}

Comment: $\mathrm{P}<0.01$, it is extremely significant, represented by **; $\mathrm{P}<0.05$, is significant, represented by *; $\mathrm{P}>0.05$, is not significant.

Table 5. Correlation coefficient of ANOVA

\begin{tabular}{cccc}
\hline Statistics project & value & Statistics project & value \\
\hline $\begin{array}{c}\text { Sample standard } \\
\text { deviation }\end{array}$ & 5.60 & R-Squared & 0.9990 \\
Mean & 469.41 & Adj R-Squared & 0.9978 \\
C V \% & 1.19 & Pred R-Squared & 0.9871 \\
\hline
\end{tabular}




\begin{tabular}{llll}
\hline Predicted sum of squares & 2974.13 & Adeq Precision & 83.779 \\
& & \\
\hline
\end{tabular}

\subsubsection{Response surface analysis}

In general, the response surface will select the highest point of the dependent variable as the apex of the response surface model, but this experiment is to explore the optimal parameters of the rice quinoa gel process and obtain the lowest hardness to leave room for changes in the subsequent gel aging hardness. Therefore, the lowest point of the surface is selected as the apex of the response surface model, and the corresponding contour lines become contour lines in this experiment.

In order to more intuitively understand the influence of each factor on each indicator, the corresponding response surface interaction diagram is obtained based on the data obtained from the regression model variance analysis, and the interactive influence of the three factors on the hardness of rice quinoa gel is explored. At the same time, etc. The low-line graph can intuitively reflect the significance of the interaction between the two factors. The circle in the contour plot indicates that there is no significant interaction, and the ellipse or pommel shape indicates that the two factors have a significant interaction [6].

The response surface graph and contour lines of the rice quinoa gel production process conditions are shown in the figure. In the response surface graph, the degree of convexity can represent the influence of different factors on the response value. It can be seen from the response surface that the curve of the graph changes the steepest

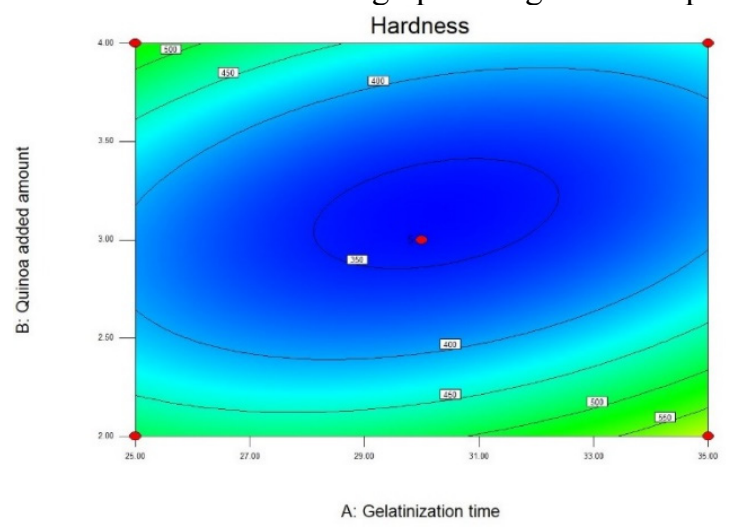

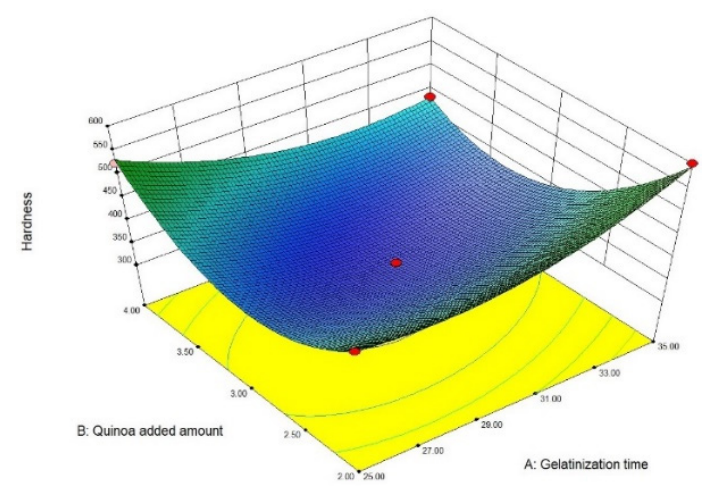

Fig 6. Response surface and contour map of the interaction between $\mathrm{A}$ and $\mathrm{B}$ on the hardness of rice quinoa gel

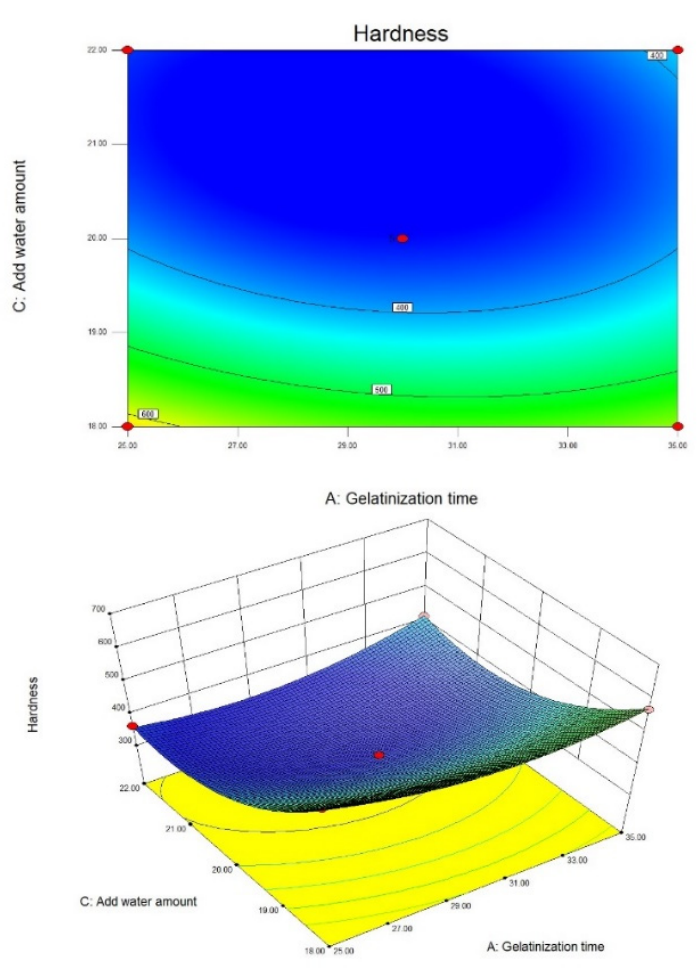

Fig 7. Response surface and contour map of the interaction between $\mathrm{A}$ and $\mathrm{C}$ on the hardness of rice quinoa gel

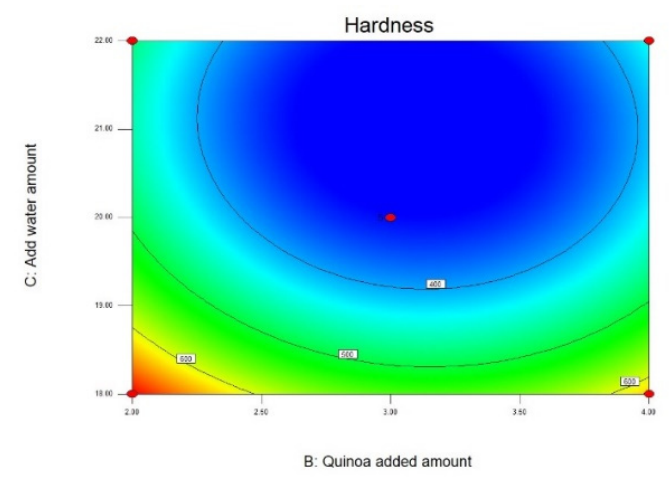




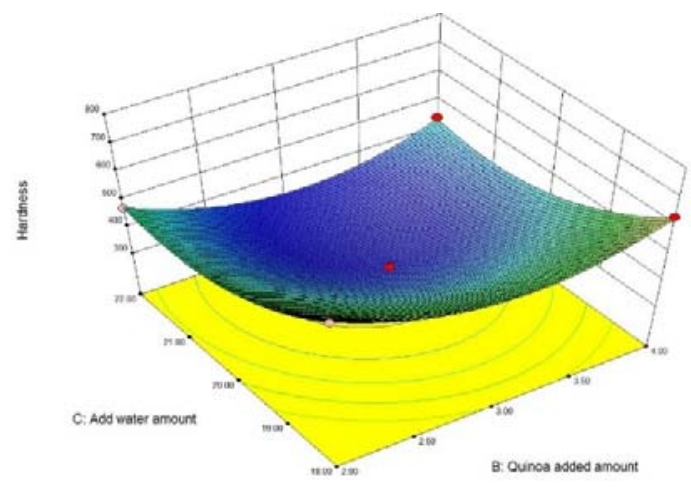

Fig 8. Response surface and contour map of the interaction between $\mathrm{B}$ and $\mathrm{C}$ on the hardness of rice quinoa gel

It can be seen from the response surface in Figure 3D that there is the lowest point of the response surface in the set range of independent variables, indicating that the selected variable range meets the requirements; the addition of quinoa has the most significant effect with the change of water addition, so it can be seen from the figure. The curve is steeper, followed by the gelatinization time and the amount of quinoa added, and the gelatinization time and the amount of water added are the most gentle, indicating that the amount of water added has the greatest impact on the hardness of the rice quinoa gel, which is consistent with the results of the hardness regression equation variance analysis ; Judging from the contour chart, the picture is nearly circular, and the effect of the ellipse is relatively obvious. Therefore, the interaction of the three factors except $\mathrm{BC}$ has a significant influence on the gel hardness, which is consistent with the results in the table. Optimize the formula.

\subsubsection{Product optimal formula and verification test}

Design Expert 8.0.6.1 was used to analyze the test results, and the optimal formula was predicted from the model: the gelatinization time was $30 \mathrm{~min}$, the amount of quinoa added was $3 \mathrm{~g} / 10 \mathrm{~g}$, the amount of water added was twice, the hardness was $343.60 \mathrm{~g}$, and the theoretical value was 0.878 . According to this ratio, three parallel experiments were carried out to take the average value, and the hardness of rice quinoa gel was obtained, which was similar to the predicted value. The product was made with strong aroma, moderate taste and no bad flavor. Therefore, it can be judged that the formula scheme of this response surface experimental design is extremely feasible and has certain guiding significance for practice.

\section{Conclusion}

In this paper, the three factors and three levels of gelatinization time (A), quinoa addition (B), and water addition (C) in the production process of rice quinoa rice noodles are used as independent variables, and the sensory score of rice quinoa gel is used as the dependent variable. According to the central law Box-Behnken experiment method, using Design-Export 8.0.6.1 software, a response surface experiment was designed. The results of the study found that the order of the influence of various factors on sensory scores is as follows; the best process conditions for the production of rice quinoa rice noodles after correction are gelatinization time $30 \mathrm{~min}$, quinoa addition amount $30 \%$, and material-to-water ratio $1: 2(\mathrm{~g} / \mathrm{mL})$. The test product of this formula has uniform color, uniform gel, strong aroma, good taste and fine mouthfeel.

\section{Acknowledgments}

This work was supported by the program of Beijing science and technology planning-Development and Brand Building of Functional Fresh-cut Vegetable Products (Z181100009318001) and Beijing Leafy Vegetables Innovation Team of Modern Agro-industry Technology Research System (BAIC07-2020).

\section{References}

1. Luca, O. Jonathan, K. Alan, O. James. 2016 Chemistry, structure, functionality and applications of rice starch, Journal of Cereal Science, 70, 291-300.

2. Xiu Yu, Liang Xiaoyan, Shi Ruichang, etc. Effect of mixed salt-alkali stress on the growth characteristics of quinoa plant and root system at seedling stage.J.Jiangsu Agricultural Sciences, 2020, 48 (4): 89-94.

3. Ren Guixing, Yang Xiushi, Mo Yang. The status quo of China's quinoa industry.J. Crop Journal, 2015 (5): $1-5$.

4. Kumpun S,Maria A, Crouzet S,et al. Ecdysteroids from Chenopodium quinoa Willd.,an ancient Andean crop of high nutritional value.J..Food Chemistry,2011,125 (4) : 1226-1234.

5. Alvarez J L,Arendt E K, Gallagher E. Nutritive value and chemical composition of pseudocereals as gluten- free ingredients.J. International Journal of Food Sciences and Nutrition,2009,60: 240-257.

6. Muralidhar R V, Chirumamila R R, Marchant R, et al. A response surface approach for the comparison of lipase production by Candida cylindracea using two different carbon sources.J. Biochemical Engineering Journal, 2001, 9(1):17-23. 